\title{
Surgical management of trabecular ventricular septal defects: The sandwich technique
}

\author{
Yoshio Ootaki, MD \\ Masahiro Yamaguchi, MD \\ Naoki Yoshimura, MD \\ Shigeteru Oka, MD \\ Masahiro Yoshida, MD \\ Tomomi Hasegawa, MD
}

\footnotetext{
From the Department of Cardiothoracic Surgery, Kobe Children's Hospital, Kobe, Hyogo, Japan.

Received for publication May 30, 2001; revisions requested July 19, 2001; revisions received March 27, 2002; accepted for publication Aug 1, 2002.

Address for reprints: Yoshio Ootaki, MD, 1-1-1 Takakuradai, Suma-ku, Kobe, Hyogo, 654-0081, Japan (E-mail: y.ootaki@nifty.ne.jp).

J Thorac Cardiovasc Surg 2003;125:508-12 Copyright ( $\odot 2003$ by The American Association for Thoracic Surgery

0022-5223/2003\$30.00+0

doi: $10.1067 / \mathrm{mtc} .2003 .56$
}

Background: Surgical closure of trabecular ventricular septal defects is difficult and often unsuccessful.

Objective: We performed closure of trabecular ventricular septal defects by sandwiching the septum between 2 polyester felt patches placed in the left ventricle and right ventricle without ventriculotomy.

Methods: Eleven patients ( 7 boys and 4 girls) underwent a sandwiching closure at a mean age of 4.7 years (range, 0.4-9.7 years) and a mean weight of $16.7 \mathrm{~kg}$ (range, 4.6-52 kg). Associated cardiac malformations were present in 9 of the 11 patients. Seven patients had undergone previous operations. The trabecular ventricular septal defects are exposed through the tricuspid valve and also from the left ventricular side through a coexisting large perimembranous ventricular septal defect or through the mitral valve through an interatrial septostomy. Two forceps, one each from the right and left ventricular side, lead a $3 \mathrm{~F}$ Nelaton catheter through the trabecular defect. An oversized circular polyester felt patch mounted on a 3-0 Nespolen suture attached to the Nelaton catheter is then passed into the left ventricle. The suture ends are then passed through a slightly smaller polyester felt patch on the right ventricular side of the septum. The Nespolen suture is then tied, thereby sandwiching the septum between the 2 patches.

Results: Time required for the procedure was less than 20 minutes in each case. There were no hospital deaths, and the postoperative course was uneventful in all patients. There was no residual shunt in 3 patients, and a minimal residual shunt was observed in 5 patients. Mild residual shunt was observed in 3 patients. Cardiac catheterization was performed 1 month postoperatively in 8 patients in whom residual shunt was noted on echocardiography. Five of 8 patients had a minimal residual shunt (pulmonary blood flow/systemic blood flow ratio $=1.0$ ). Three patients had a residual shunt (pulmonary blood flow/systemic blood flow ratio $=$ 2.0, 1.6, and 1.2). The patient with a pulmonary blood flow/systemic blood flow ratio of 2.0 had a "Swiss cheese" ventricular septal defect, and a residual shunt remained around the patch. However, the residual shunt decreased to a pulmonary blood flow/systemic blood flow ratio of 1.6 at examination 16 months postoperatively. Echocardiography showed that the residual shunt had also decreased in another 2 patients.

Conclusions: We conclude that the sandwich technique is safe and easy. Even in cases with a residual shunt present, the shunt is expected to decrease as time passes. Further experience and longer follow-up of these patients are necessary to conclude whether this technique is applicable to neonates and young infants. 


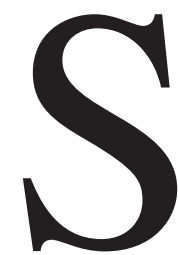

urgical difficulties for closure of multiple ventricular septal defects (VSDs) were mainly encountered for trabecular VSDs. ${ }^{1}$ Improved techniques for repair of this challenging pathology while avoiding a right or left ventriculotomy were recently described. ${ }^{2-4}$ However, complete repair of such defects remains associated with high morbidity and mortality because of residual VSDs and postoperative myocardial dysfunction. To address these potential drawbacks, we closed trabecular VSDs by sandwiching the septum between the 2 polyester felt patches placed in the left ventricle and right ventricle without ventriculotomy. ${ }^{5}$

\section{Patients and Methods}

From November 1998 to March 2001, 11 consecutive patients with a diagnosis of trabecular VSD underwent surgical repair at the Department of Cardiothoracic Surgery, Kobe Children's Hospital, Japan. There were 7 boys and 4 girls, with a mean age of 4 years and 7 months (range, 4 months to 9 years and 7 months) and a mean weight of $16.7 \mathrm{~kg}$ (range, 4.6-52.0 kg). The preoperative diagnosis and surgical anatomic findings are summarized in Table 1.

Associated cardiac malformations were present in 9 of the 11 patients. Seven of 11 patients had previous cardiac operations to increase or decrease pulmonary blood flow. One patient had associated pulmonary stenosis to restrict pulmonary blood flow, and 3 patients showed pulmonary hypertension; their mean age was 9 months.

\section{Anatomic Definitions}

Eight patients had 2 VSDs, and 3 patients had 3 VSDs. Surgical anatomy of the trabecular VSD is based on the works of Serraf and colleagues. ${ }^{6}$ The trabecular septomarginalis is an important landmark for classification of the trabecular VSD. The trabecular septomarginalis is a muscular structure that lies on the right surface of the muscular septum. There are 3 main components of

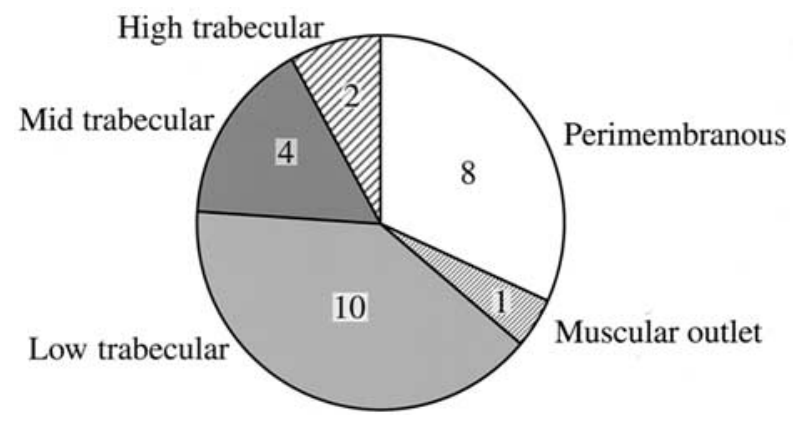

Figure 1. Location of VSDs.

the trabecular septum: the high trabecular septum, the midtrabecular septum, and the low trabecular septum.

Low trabecular septal defects are difficult to close, and closure has previously been achieved with a ventriculotomy. In our case, $8(73 \%)$ of 11 patients had low trabecular septal defects. The location of the VSDs is depicted in Figure 1.

\section{Surgical Technique for Trabecular VSDs}

The surgical technique for trabecular VSDs is shown in Figure 2. Total cardiopulmonary bypass is established between the ascending aorta and both vena cavae. Myocardial protection is achieved by using moderate systemic hypothermia, cold blood antegrade cardioplegia repeated every 10 minutes, and topical cooling. A longitudinal right atriotomy is performed, and stay sutures were placed on the inner wall of the right atrium to evert the tricuspid valve. A longitudinal atrioseptotomy was performed in 5 of 11 patients. Infundibulotomy was performed in 4 patients to repair associated cardiac malformations.

The trabecular septum is exposed through the tricuspid valve. We negotiate a right-angled forceps to the right-sided trabecular VSD through the tricuspid valve. We also negotiate another forceps to the left-sided trabecular VSD through another perimembranous VSD. In case another large VSD is absent, a longitudinal

TABLE 1. Summary of data on patients undergoing sandwich repair of trabecular VSDs

\begin{tabular}{|c|c|c|c|c|c|}
\hline Patient no. & Age (y) & Sex & Morphology & Another VSD & Previous operation \\
\hline 1 & 9.7 & $M$ & DORV,PA,mVSDs(1) & Perimembranous & Bilateral BT \\
\hline 2 & 7.1 & $\mathrm{~F}$ & mVSDs(1),PFO & Perimembranous & PAB \\
\hline 3 & 6.1 & $\mathrm{~F}$ & DORV,PS,mVSDs(2) & Perimembranous & Bilateral BT \\
\hline 4 & 5.8 & $\mathrm{M}$ & AVSD,TF,mVSDs(1) & Perimembranous & rt.BT \\
\hline 5 & 0.4 & $\mathrm{M}$ & ASD,mVSDs(1) & Perimembranous & - \\
\hline 6 & 0.10 & $\mathrm{M}$ & MR,mVSDs(1) & Muscular outlet & - \\
\hline 7 & 6.5 & $\mathrm{~F}$ & $\operatorname{mVSDs}(2)$ & - & PAB \\
\hline 8 & 3.0 & $M$ & PS,mVSDs(1) & Perimembranous & - \\
\hline 9 & 5.6 & $\mathrm{~F}$ & mVSDs(3),PFO,PDA & - & PAB \\
\hline 10 & 5.1 & $\mathrm{M}$ & mVSDs(2),PFO,PDA & Perimembranous & PAB \\
\hline 11 & 1.1 & $\mathrm{M}$ & $\operatorname{mVSDs}(1)$ & Perimembranous & - \\
\hline
\end{tabular}

Numbers in parentheses indicate the number of trabecular VSDs.

DORV, Double-outlet right ventricle; $P A$, pulmonary atresia; $m V S D S$, multiple VSDs; $P F O$, patent foramen ovale; $P S$, pulmonary stenosis; $A V S D$, atrioventricular septal defect; $T F$, tetralogy of Fallot; $A S D$, atrial septal defect; $M R$, mitral regurgitation; $P D A$, patent ductus arteriosus; $B T$, Blalock-Taussig shunt; $P A B$, pulmonary artery banding. 


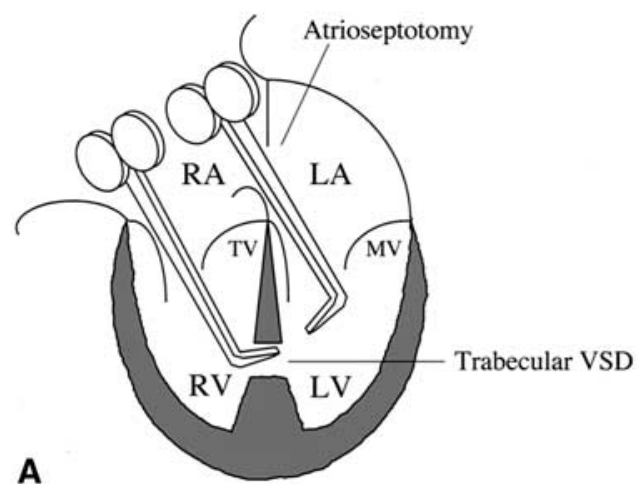

B
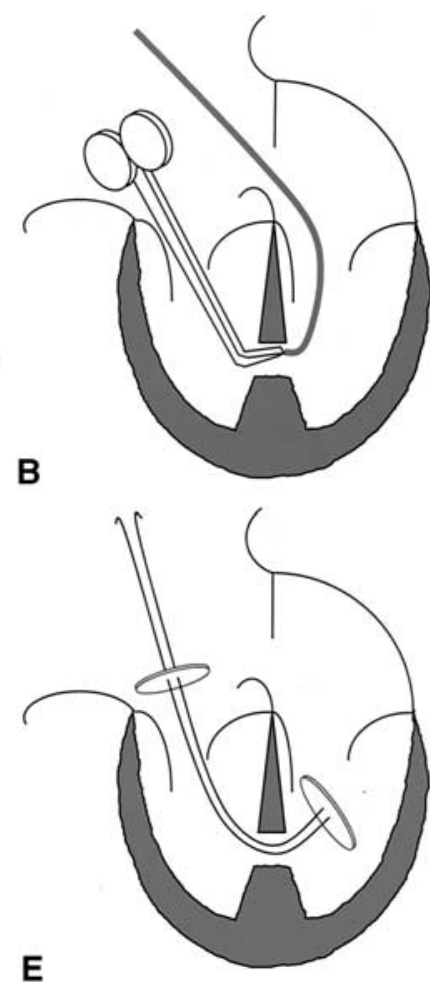

D

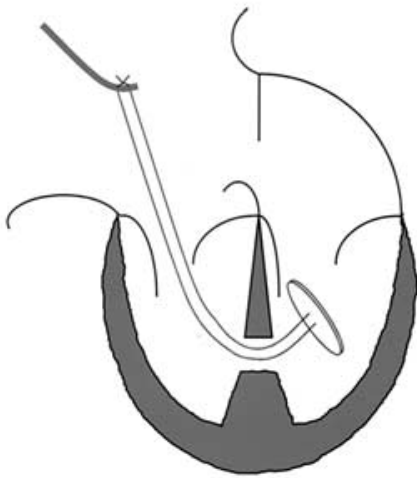

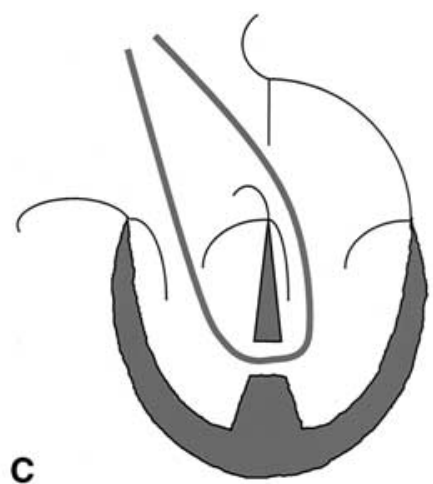

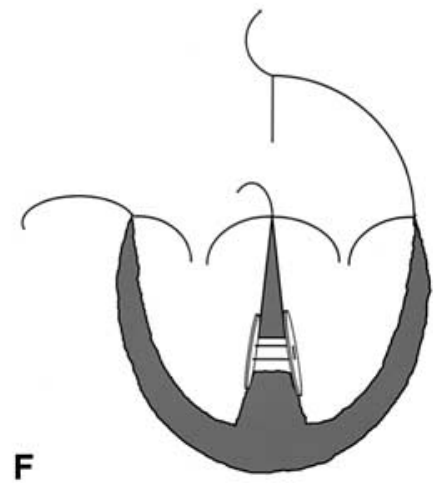

$E$

Figure 2. A, Negotiating 2 forceps through the tricuspid valve and mitral valve permits the opening of the trabecular VSD. B and C, Two forceps lead the Nelaton catheter through the trabecular VSD. D, The Nelaton catheter leads an oversized (at least 8-10 mm larger than the estimated size of the VSD) circular polyester felt patch mounted on a 3-0 Nespolen suture into the left ventricle through the mitral valve. $E$, The right-sided patch was sewn to the 3-0 Nesporen suture. F, The Nespolen suture is then tied firmly, thereby sandwiching the septum between the 2 polyester felt patches. $L V$, Left ventricle; $R V$, right ventricle; $L A$, left atrium; $R A$, right atrium; $T V$, tricuspid valve; $M V$, mitral valve. atrioseptotomy is performed to insert the forceps into the left ventricle. This permits the right side of the septum to be probed gently to locate the right-sided openings and the (fewer) left-sided openings. Two forceps lead the $3 \mathrm{~F}$ Nelaton catheter through the trabecular VSD. The Nelaton catheter leads an oversized (at least 8-10 mm larger than the estimated size of the VSD for the safety margin) circular polyester felt patch (Bard, Inc, Haverhill, Mass) mounted on a 3-0 Nespolen suture (Azwell, Inc, Osaka, Japan) through the large VSD or the mitral valve. The suture ends are then passed through a slightly smaller (at least 6-7 $\mathrm{mm}$ larger than the estimated size of the VSD) polyester felt patch on the right ventricular side of the septum. The Nespolen suture is then tied, thereby sandwiching the septum between the 2 polyester felt patches. We could confirm that the mitral valve apparatus was not impaired through the mitral valve itself.

\section{Results}

All patients had successful sandwich repair of trabecular VSDs, and 15 VSDs were closed by using this technique. The mean size of the trabecular VSD was $5.4 \mathrm{~mm}$. The mean size of the left-sided patch was $14.2 \mathrm{~mm}$, and that of the right-sided patch was $11.7 \mathrm{~mm}$. The left-sided patch did not impair the apparatus of the mitral valve in any case. The aortic crossclamp time was $132 \pm 64$ minutes (range, 68252 minutes) because of the repair of associated cardiac lesions, but the procedural time for closure of the trabecular VSDs was less than 20 minutes in each case.

There were no hospital deaths. The postoperative course was uneventful in all patients. Electrocardiography showed sinus rhythm in all patients, with complete right bundle block in 3 patients. Postoperative echocardiography did not show any change in left ventricular function. There was no residual shunt in 3 patients, and a minimal residual shunt was observed in 5 patients. Mild residual shunt was observed in 3 patients. Postoperative catheterization was performed 1 month postoperatively in 8 patients in whom residual shunt was noted on echocardiography. Five of 8 patients had a minimal residual shunt (pulmonary blood flow/systemic blood flow ratio $[\mathrm{Qp} / \mathrm{Qs}]=1.0$ ). Three patients had a residual shunt $(\mathrm{Qp} / \mathrm{Qs}=2.0,1.6$, and 1.6, respectively). Patient 1 had a "Swiss cheese" VSD, and residual shunt remained around the patch. However, resid- 

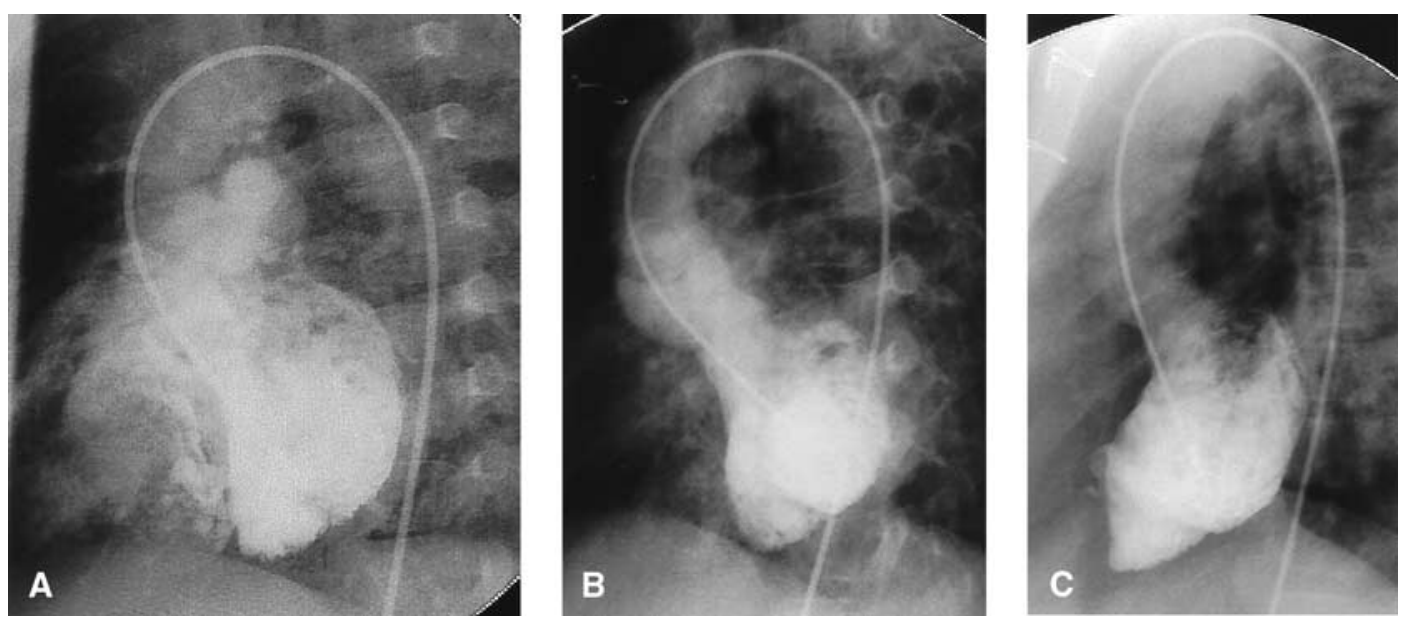

Figure 3. Left ventriculography of patient 3. A, Preoperative left ventriculography showed perimembranous and trabecular VSDs. B, Left ventriculography 1 month postoperatively showed a minimal residual shunt. C, Left ventriculography 14 months postoperatively did not show any residual shunt.

ual shunt decreased to a Qp/Qs of 1.6 at recatheterization 16 months postoperatively. In all but patient 3, pulmonary arterial pressure was in the normal range postoperatively. Patient 3 had mild pulmonary hypertension and minimal residual shunt postoperatively, but residual shunt disappeared 14 months postoperatively (Figure 3 ).

Patients were followed up clinically and echocardiographically for a mean of $20 \pm 5.4$ months. There were no late deaths or reoperations, except in patient 1 . Electrocardiography of patient 1 demonstrated premature ventricular contractions preoperatively and postoperatively, and the patient was found to be apneic and pulseless at school approximately 2 years later. On postmortem examination, the trabecular VSD was completely closed, with proliferated intima and fibrous tissue around the polyester felt patch.

\section{Discussion}

Despite advances in preoperative diagnosis, improved surgical techniques, and postoperative care, there is still significant morbidity and mortality in the treatment of patients with trabecular VSDs. The moderator band and the multiple trabeculations beneath it create a multiperforated wall that hides trabecular VSDs on the transtricuspid view. For this reason, apical left ventriculotomy was introduced and rapidly adopted as the most effective approach for surgical closure of trabecular VSDs. However, late complications, including apical aneurysm or dyskinesis, have created the need for a safer approach. Before we started closing the trabecular VSD with the sandwich technique, it had been our strategy to use pulmonary artery banding or systemicto-pulmonary shunt to defer the time of definitive repair as late as possible. We had to close trabecular VSDs in 2 patients in the past: one was a 6-year-old patient with tetralogy of Fallot and low trabecular multiple VSDs, and the other was a 2-year-old patient with a large VSD, mitral regurgitation, and multiple low trabecular VSDs who had extended aortic arch anastomosis and pulmonary artery banding for coarctation complex and arch hypoplasia in the newborn period. Both of these patients had stormy postoperative courses and longstanding left-heart failure, resulting in late death caused by chronic renal failure in the latter patient. Kitagawa and associates ${ }^{2}$ described the technique of removing a section of the moderator band as an approach to defects in the midtrabecular septum. Although this avoided the need for left ventriculotomy in most patients, late follow-up data concerning right and left ventricular and atrioventricular valve function are not yet available.

Transcatheter device implantations $\mathrm{s}^{7,8}$ seem to be useful to close high anterior or apical defects; however, the devices are not yet available for use in Japan. Moreover, devices must be placed intraoperatively in infants because of the size and stiffness of the catheters.

Nine of the 11 patients in our series also had associated malformations requiring surgical intervention. Trabecular VSDs were closed by using the sandwich technique at the time of definitive repair for associated defects, sparing operative time considerably.

Combined color-coded echocardiography and angiography have proved to be a reliable means of obtaining an accurate preoperative diagnosis ${ }^{9}$; however, it remains difficult to ascertain whether all trabecular VSDs are diagnosed, particularly when there is a nonrestrictive defect. In our case we could detect additional trabecular VSDs in 4 patients at surgical intervention in addition to the one suspected preoperatively. Preoperative accurate diagnosis is critical to treat such patients.

Despite improved integrated treatment of multiple VSDs 
between surgical and interventional techniques, there is still significant concern regarding the optimal treatment of a trabecular VSD. This has led to considerable controversy regarding the optimal management of these patients. Banding of the pulmonary artery to protect the pulmonary vascular bed and to relieve symptoms of congestive heart failure has traditionally been used in an effort to delay definitive repair, as was the former treatment strategy in our institution. This staged approach might allow time for or even promote spontaneous closure of some of the defects, possibly increasing the likelihood of successful surgical outcome. However, the deleterious effects of biventricular muscular and fibrous hypertrophy and long-lasting hypoxia caused by this approach, leading to arrhythmic events and chronic heart failure in the future, is certainly undeniable. Since we invented the sandwich technique, none of the patients had pulmonary artery banding, and we closed the trabecular VSD safely, even in a 4-month-old infant. In the case of an even smaller baby with trabecular VSDs, it is not indisputable that a large sandwich patch might impair the distensibility of the left ventricle. Further experience and longer follow-up of these patients are needed to conclude whether this technique can be used consistently in neonates and young infants.

\section{References}

1. Kirklin JK, Castaneda AR, Keane JF, Fellows KE, Norwood WI. Surgical management of multiple ventricular septal defects. J Thorac Cardiovasc Surg. 1980;80:485-93.

2. Kitagawa T, Durham LA III, Mosca RS, Bove EL. Techniques and results in the management of multiple ventricular septal defects. J Thorac Cardiovasc Surg. 1998;115:848-56.

3. Mace L, Dervanian P, Bret EL, Folliguet TA, Lambert V, Losay J, et al. "Swiss cheese" septal defects: surgical closure using a single patch with intermediate fixings. Ann Thorac Surg. 1999;67:1754-9.

4. Stellin G, Padalino M, Milanesi O, Rubino M, Casarotto D, Van Praagh R, et al. Surgical closure of apical ventricular septal defects through a right ventricular apical infundibulotomy. Ann Thorac Surg. 2000;69:597-601.

5. Yamaguchi M, Yoshimura N, Oka S, Ootaki Y, Yoshida M. Closure of muscular VSD by a sandwiching method via a coexisting larger VSD or an interatrial septostomy. Presented at the 3rd World Congress of Pediatric Cardiology and Cardiac Surgery; 2001. p. 227.

6. Serraf A, Lacour-Gayet F, Bruniaux J, Ouaknine R, Losay J, Petit J, et al. Surgical management of isolated multiple ventricular septal defects. J Thorac Cardiovasc Surg. 1992;103:437-43.

7. Fishberger SB, Bridges ND, Keane JF, Hanley FL, Jonas RA, Mayer JE, et al. Intraoperative device closure of ventricular septal defects. Circulation. 1993;88:205-9.

8. Amin Z, Berry JM, Foker JE, Rocchini AP, Bass JL. Intraoperative closure of muscular ventricular septal defect in a canine model and application of the technique in a baby. $J$ Thorac Cardiovasc Surg. 1998; 115:1374-6

9. Ludomirsky A, Huhta JC, Vick W III, Murphy DJ, Danford DA, Morrow WR. Color doppler detection of multiple ventricular septal defects. Circulation. 1986;74:1317-22. 